\title{
Das Alter des Sylter Kaolinsandes
}

\author{
Von Richard Weyl, Kiel (I u. II), Ulrich Rein, Krefeld (III) \\ und Marlies Teichmüller, Krefeld (IV) \\ Mit $2 \mathrm{Abb}$.
}

$\mathrm{Zusammenfassung.} \mathrm{Aus} \mathrm{den} \mathrm{Profilen} \mathrm{des} \mathrm{Kaolinsandes} \mathrm{am} \mathrm{Morsum-Kliff} \mathrm{und} \mathrm{am}$ Roten Kliff auf Sylt wurden 2 Braunkohlenflöze von je $1 \mathrm{~m}$ Mächtigkeit pollenstratigraphisch und die Begleitschichten sedimentpetrographisch untersucht. Bei einem Vergleich der Pollenzusammensetzung mit anderen Horizonten aus dem mittleren Pliozän bis zum mittleren Pleistozän in Nordwestdeutschland zeigt sich eine gute Ubereinstimmung mit der oberpliozänen Reuverstufe. Auch alle vorhergehenden pollenanalytischen Untersuchungen von Braunkohlenproben aus den Kaolinsanden kamen zu dem gleichen Rंesultat. Es bleibt somit bei der Stellung des Kaolinsandes im Oberpliozän. Daher muß die von D. Wirtz und H. Illies vorgenommene Neudatierung des Tertiärprofils am Morsum-Kliff als hinfällig gelten. Auch sind die Überlegungen über die Lage der Plio-Pleistozängrenze auf der Insel Sylt zu revidieren. Die Verwitterung der Kaolinsande hat mit Sicherheit vor der Elster-Vereisung (Mindel) und aller Wahrscheinlichkeit nach im allerjüngsten Pliozän stattgefunden. Im Profil des Roten Kliffs ist der Schwermineralbestand in den tonigen Lagen vor einer nachträglichen Verwitterungsauslese geschützt geblieben. Damit sind gewisse Unterschiede in der Mineral-Assoziation zu erklären. Zum Alter des Limonitsandsteins im Sylter Profil kann auf Grund der vorliegenden Untersuchung keine Stellung genommen werden.

$\mathrm{Su} \mathrm{mm}$ a r y. Pollen-stratigraphical studies of 2 brown coal seams of about 1 metre thickness have been made. The seams occur in the profils of Kaolin Sand in the Morsum Cliff and in the Red Cliff at Sylt. The accompanying strata have been examined by sedimentary petrographic methods. After comparing the pollen composition here with that of other horizons from the Middle Pliocene to the Middle Pleistocene, a large agreement has been found with the flora of the Upper Pliocene Reuver Stage. All the pollen analyses of the Kaolin Sand samples gave the same result. The age of these sands thus remains Upper Pliocene. This means that the conclusions of $\mathrm{D}$. Wirtz und $\mathrm{H}$. Illies as to the age of the Tertiary profile at Morsum Cliff must be revised. Their ideas of the position of the Pliocene-Pleistocene boundary at Sylt should also be reconsidered. The weathering of the Kaolin Sand occured certainly before the Elster Glaciation (Mindel) and most probably in the very youngest Pliocene. In the profile at Red Cliff the heavy mineral content of the clay deposits has remained, due to subsequent preferential weathering effects. Certain differences in the mineral asșociations may be explained by this fact. On the basis of the above examinations no age can be given for the limonitic sandstone in the Sylt profile.

\section{Einle itung und Fragestellung}

In einer Reihe von Arbeiten haben WIRTz und Illies (WIRTz 1949, WIRTz \& ILlies 1951a, 1951b) versucht, das bekannte Jungtertiär-Profil des Morsumkliffs auf Sylt neu zu datieren, um damit ein Standardprofil für die Festlegung der Plio-Pleistozängrenze in Nordwestdeutschland zu geben. WIRTz (1949) kam durch eine Revision der Fauna des Limonitsandsteins zu der Auffassung, dạß dieser dem höchsten Pliozän angehöre und mit dem Waltonian Ostenglands zu parallelisieren sei. Auf Grund dieser Einstufung und lithologischer Erwägungen entwickelten sodann WIRTz \& II.LIES (1951a, 1951b) die Vorstellung, daß die über dem Limonitsandstein folgende sandige Serie, die sie in einen ästuarinen Feinsand und den hangenden Kaolinsand untergliederten, das tiefere Alt-Pleistozän vertrete und mit der englischen Schichtfolge vom jüngeren Red Crag bis zum Cromer Forest Bed zu parallelisieren sei.

Gegen diese Deutung des Profiles vom Morsumkliff wurden jedoch Bedenken laut: Woldstedt (1950) mahnte auf Grund der Pollenuntersuchungen Thomson's (1948) an Braunkohlen aus dem Hangenden des Sylter Limonitsandsteines zur Vorsicht und forderte neue Untersuchungen, um eine Klärung der Frage der ältesten Kaltzeit und eine: ältesten Interglazialzeit in Nordwestdeutschland herbeizuführen. DIETz \& HECK (1952b) 
verwiesen auf die Pollenuntersuchungen Thiergart's (1944) an einem Saprohumulith im höheren Kaolinsand des Roten Kliffs und auf die von v. d. BreLIE \& REIN (1951) ausgeführten Pollenuntersuchungen an Braunkohlengeröllen des Nord- und Weststrandes der Insel, die auf ein pliozänes Alter der Braunkohlen hinweisen, so daß sie keinen Grund sahen, die frühere Gliederung des Sylter Tertiärs aufzugeben, derzufolge der Kaolinsand dem Oberpliozän angehört. WEYL (1952) äußerte auf Grund der sehr starken, in situ erfolgten Verwitterungserscheinungen im Kaolinsand Bedenken gegen ihr pleistozänes Alter, da diese kein Analogon in anderen Ablagerungen entsprechenden Alters hätten.

Im gleichen Jahre legte Thomson (1952) das Ergebnis der pollenanalytischen Untersuchung an einer Braunkohlenprobe vom Roten Kliff im Archiv des Amtes für Bodenforschung, Landesstelle für Nordrhein-Westfalen nieder, die er dankenswerterweise neuerdings auch der Offfentlichkeit zugänglich gemacht hat (TномsоN 1955). Hierin bestätigt er das Ergebnis seiner früheren Untersuchungen, denen gemäß die Kohlen der Reuver-Stufe angehören.

Eine Klärung der Altersfrage des Sylter Kaolinsandes erscheint daher nur möglich, wenn neues Beobachtungsmaterial bereitgestellt wird, das vor allem aus einer pollenanalytischen Untersuchung der dem Kaolinsand eingelagerten Braunkohlenflöze zu erhoffen war. Diese Flöze sind zwar sowohl vom Morsumkliff wie vom Roten Klifi altbekannt, liegen jedoch meist unter Abrutschmassen verborgen, so daß sie auch WIRTz. \& ILliEs zur Zeit ihrer Untersuchungen nicht zugänglich waren. Infolge der Frühjahrsstürme waren sie jedoch im März des Jahres 1954 angeschnitten und konnten anläßlich einer Exkursion des Geol.-Pal. Institutes der Universität Kiel aufgegraben werden. ${ }^{1}$ ) Damit war es möglich, frisches, dem Schichtenverband entnommenes Material der pollenanalytischen Untersuchung zuzuführen, über die in Abschnitt III berichtet wird.

\section{Profilbeschreibung und Sedimentologie \\ der Begleitschichten}

Das am Mors umkliff freigelegte Braunkohlenflöz steht ca. $300 \mathrm{~m}$ westlich des „Pegels“ und $100 \mathrm{~m}$ W des sog. Walfischrückens an. Es war an der Basis des bewachsenen toten Kliffs auf eine Länge von $35-40 \mathrm{~m}$ freigelegt. Zum Erfassen des Liegenden wurde ein Schurf angelegt. Das Profil stellt sich wie folgt dar:

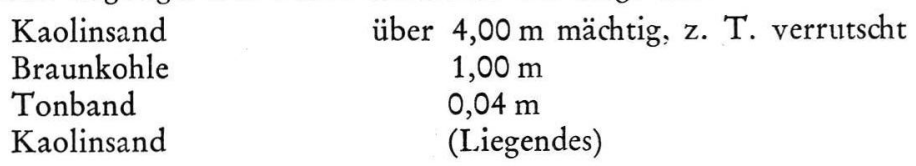

Am Roten K liff liegt das Flöz am Kliffuß an der auf der Geologischen Karte (DIETz \& Heck 1952a) durch die Signatur "plfs" gekennzeichneten Stelle. Es war auf ca. $30 \mathrm{~m}$ Länge aus dem Schuttfuß des. Kliffs freigespült und konnte durch einen Schurf bis auf das Liegende erschlossen werden. Das Profil steilt sich wie folgt dar:
Geschiebelehm (saaleeiszeitlich)
Kaolinsand
grauer Ton
Braunkohlenflöz

$$
\begin{array}{r}
\text { ca. } 9,00 \mathrm{~m} \\
\text { ca. } 6,00 \mathrm{~m} \\
0,10 \mathrm{~m} \\
1,00 \mathrm{~m}
\end{array}
$$

1) Magnifizenz HofmanN sei bei dieser Gelegenheit fuir die finanzielle Unterstützung der Exkursion gedankt. An den Aufgrabungen beteiligten sich die Herren cand. geol. ERKwOH, Hofmann und Pieles, denen für ihre Mitwirkung ebenso gedankt sei wie Herrn cand. geol. HoFmann für die Hilfe bei der sedimenpetrographischen Untersuchung der Sande.

Das Ergebnis vorliegender Arbeit wurde im September 1954 auf der Tagung der Deutschen Quartärvereinigung von U. REIN vorgetragen. 


$\begin{array}{ll}\text { brauner Ton } & 0,10 \mathrm{~m} \\ \text { gelber Sand } & 0,08 \mathrm{~m} \\ \text { gelber Ton } & 0,06 \mathrm{~m} \\ \text { gelber Sand } & 0,04 \mathrm{~m} \\ \text { weißer Kaolinsand } & \text { Liegendes }\end{array}$

Zur näheren Kennzeichnung der liegenden und hangenden Begleitschichten der Flöze wurden Schwermineraluntersuchungen vorgenommen, da aus dem Zustand der Schwermineralgesellschaft Zweifel an der Einstufung der Kaolinsande in das Pleistozän erwachsen waren (WEYL 1952).

Frühere Untersuchungen hatten gezeigt, daß die Kaolinsande eine an instabilen Mineralen verarmte Schwermineralassoziation aufweisen (WETZEL 1931), daß aber in manchen Horizonten, vor allem solchen, die durch ihre Zusammensetzung oder Verkittung den Verwitterungslösungen weniger zugänglich gewesen waren, die volle Schwermineralassoziation der fennoskandischen Schüttungen mit Hornblende, Epidot, metamorphen und stabilen Mineralen enthalten ist. Hieraus war die Vorstellung entwickelt worden (WEYL 1952), daß die Verarmung der Kaolinsande an Schwermineralen auf eine sehr intensive Verwitterung in situ zurückzuführen sei, die schwer mit den klimatischen Verhältnissen des Pleistozäns in Übereinstimmung gebracht werden kann, zumal sie in anderen ältest-pleistozänen Sedimenten Mitteleuropas vermißt wurde.

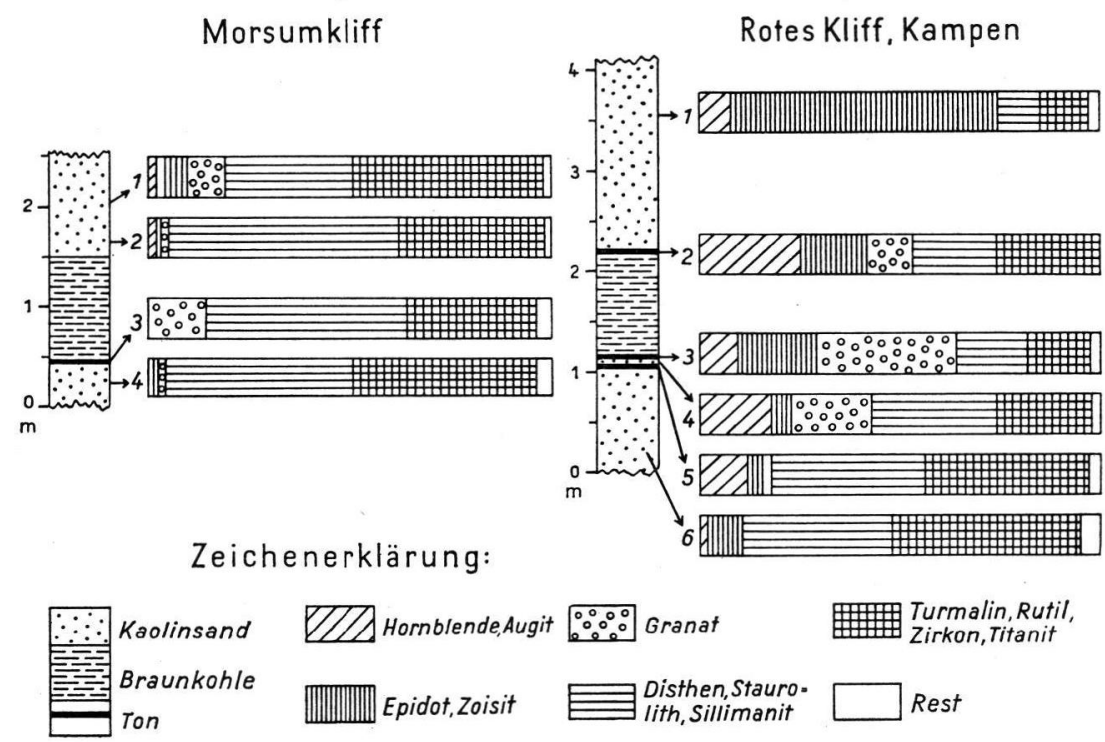

Abb. 1. Profil der Sylter Braunkohlenvorkommen und Schwermineraldiagramm ihrer Begleitschichten.

In Übereinstimmung mit einer späteren Untersuchung CвоммеLIN's (1954) war festgestellt worden, daß Granat in den Kaolinsanden Sylts in nur geringen Mengen enthalten sei, wodurch sich diese von den Vorkommen im Raume der Niederelbe und Oldenburgs unterschieden.

Die Schwermineralassoziationen in den Sanden über und unter dem B raunk o hle $\mathrm{nflöz} \mathrm{des} \mathrm{Morsumkliffs} \mathrm{entsprechen} \mathrm{weitgehend} \mathrm{dem} \mathrm{bisherigen} \mathrm{Bilde} \mathrm{und}$ sind durch das Vorherrschen der metamorphen Minerale Disthen, Staurolith, Sillimanit und der stabilen Minerale Turmalin, Rutil, Zirkon und Titanit charakterisiert. Abweichend von dem bisherigen Bilde ist ein Granatgehalt bis zu 13\% der durchsichtigen Schwerminerale. 
Ganz anders stellt sich die Schwermineralverteilung in dem Profil von $\mathrm{K}$ ampen dar: Während der $40 \mathrm{~cm}$ unter dem Flöz entnommenene Sand die gleiche Assoziation metamorpher und stabiler Minerale aufweist wie das Profil am Morsumkliff, finden wir in den Tonen über und unter dem Flöz sowie in der zwischen den beiden Tonbänkchen liegenden Sandschicht reichlich Hornblende, Granat und Epidot, während der $2 \mathrm{~m}$ über dem Flöz entnommene Sand durch eine starke Epidotvormacht gekennzeichnet ist. Wie sind diese verschiedenartigen Schwermineralassoziationen zu deuten?

Bei der schon durch ihren Geröllbestand ausgewiesenen Herkunft der Kaolinsande aus Fennoskandia und dem langen Transportweg des Materiales mit der Möglichkeit weitgehender Homogenisierung des Mineralbestandes dürfte die Annahme verschiedener "Schüttungen" erhebliche Schwierigkeiten bereiten, zumal die untersuchten Sedimente im gleichen Profil unmittelbar übereinander liegen. Eine mechanische Sonderung im fließenden Wasser auf Grund des spezifischen Gewichtes, die sich in den altbekannten Erzseifen des Kaolinsandes anzeigt, könnte für unterschiedliche Schwermineralgesellschaften verantwortlich gemacht werden, wenn nicht ausgerechnet diejenigen Minerale gemeinsam zunähmen, die sich durch unterschiedliches Sortierungsverhalten auszeichnen: Hornblende und Granat.

Einen Hinweis auf das Zustandekommen der verschiedenen Mineralassoziationen gibt der Zustand der Hornblenden, ausgedrückt in einem Ätz- und Bleichwert im Sinne von WEYL 1952: In den hornblendearmen Schichten sind die wenigen vorhandenen Hornblenden stark geätzt und weisen die charakteristischen Hahnenkämme auf, auch überwiegen farblose und zart gefärbte Körner. In den hornblendereichen Lagen dagegen sind die Hornblenden dunkel gefärbt und lassen keine oder nur sehr schwache Atzspuren erkennen. Auffallend ist fernerhin, daß ausgerechnet die Tone und die von Tonen eingeschlossene Sandlage reichlich Hornblende und sogar frische Pyroxene enthalten, also diejenigen Minerale, die nach der Stabilitätsreihe der Oberflächenverwitterung (WIESENEDER 1953) zu den instabilsten Mineralen zu rechnen sind.

Es darf also wohl in Übereinstimmung mit früheren Beobachtungen und Feststellungen an den Kaolinsanden anderer Fundorte (WEYL 1952, 1953) angenommen werden, $\mathrm{daß}$ in dem Kampener Profil in den tonigen Lagen der Schwermineralbestand vor einer nachträglichen Verwitterungsauslese geschützt blieb und sich so die Garnitur eines „Vollsandes“ im Sinne WIEsEneder's (1953) erhielt. Die Herausbildung eines Epidotmaximums im hangenden Sande steht nach den Beobachtungen Wieseneder's und eigenen Feststellungen mit dieser Deutung in Einklang, da es auf Grund der relativen Stabilität des Epidotes zur Herausbildung mehr oder minder reiner Epidotassoziationen kommen kann.

Auf eine Schwierigkeit dieser Deutung sei jedoch hingewiesen: Während bei der Schwermineralauslese durch Verwitterung der Granat üblicherweise mehr oder minder stark ausgeprägte Ätzformen aufweist, ist er in den vorliegenden Proben bis auf Ätzgruben bemerkenswert frisch. Der hohe Granatgehalt ergänzt auf jeden Fall das bisherige Bild der Sylter Schwermineralassoziation, da er bisher nicht bekannt war (CromMELIN 1954).

Der Zeitpunkt der Verwitterung des Kaolinsandes ergibt sich aus dem Zustande der nächstjüngeren Ablagerung, der in die Elstervereisung gestellten Sande des Roten Kliffs: Schon WETzEL (1937) wies darauf hin, daß in ihm instabile Minerale reichlich enthalten sind, und die Nachuntersuchung einer neu entnommenen Probe ergab einen erheblichen Anteil völlig frischer dunkler Hornblenden. Demnach hat also die den Kaolinsand charakterisierende Verwitterung vor der Ablagerung der elstereiszeitlichen Kiese stattgefunden. Eine weitere Einengung der Verwitterungszeit ist allerdings nach dem vorliegenden Material nicht möglich. 
Tabelle 1

Schwerminerale in den Begleitschichten der Sylter Braunkohlenflöze

\begin{tabular}{|c|c|c|c|c|c|c|c|c|c|c|c|c|c|c|c|c|c|c|c|c|c|}
\hline & & 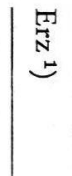 & 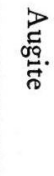 & 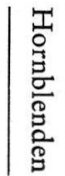 & 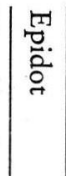 & 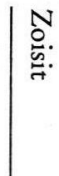 & 垔 & & 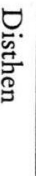 & 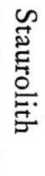 & 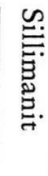 & & & & 苞 & $\mid$ & & & 莹 & 苞 & \\
\hline
\end{tabular}

I. Morsumkliff

M 1) Kaolinsand,

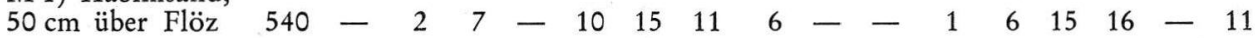

$\begin{aligned} & \text { M 2) Kaolinsand, } \\ & 10 \mathrm{~cm} \text { über Flöz }\end{aligned} 210-\begin{array}{lllllllllllllll} & & & 2 & - & 2 & 25 & 23 & 9 & - & - & 1 & 9 & 5 & 14\end{array}$

B raunkohle

$100 \mathrm{c} \mathrm{m}$

M 3) sandiger Ton $350-1-\begin{array}{llllllllllllll}1 & - & 13 & 25 & 16 & 9 & 1 & 2 & 1 & 9 & 7 & 9 & - & 7\end{array}$

M 4) Kaolinsand

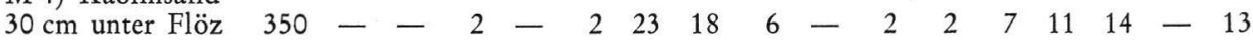

II. Rotes $\mathrm{Kliff}$

bei Kampen

K 1) Kaolinsand

$2 \mathrm{~m}$ über Flöz $115 \quad 1 \quad 7 \quad 64 \quad 2+7+\begin{array}{lllll}7 & 3 & 1 & 1\end{array}$

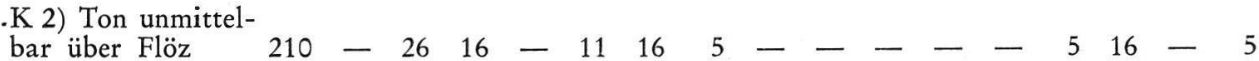

Braunkohle

$100 \mathrm{~cm}$

K 3) Brauner Ton

unmittelb. unt. Flöz $\begin{array}{lllllllllllllllllll}200 & 2 & 8 & 20 & - & 34 & 5 & 10 & 2 & 2 & - & - & 13 & 2 & 2\end{array}$

K 4) Sandlinse

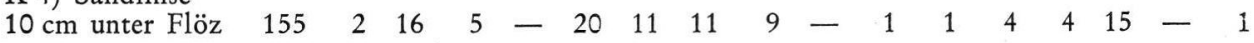

K 5) Unterer

gelber Ton

K 6) Kaolinsand

$40 \mathrm{~cm}$ unter Flöz $205-\begin{array}{lllllllllllllll} & 2 & 5 & 2 & 1 & 19 & 9 & 9 & - & 4 & 3 & 23 & 10 & 10 & -\end{array}$

Tabelle 2

Atz- und Bleichwerteder Hornblendeim Kampener Profil

$\begin{array}{lcc} & \text { Ätzwert } & \text { Bleichwert } \\ \text { K } 1 & 28 & 46 \\ \text { K } 2 & 10 & 10 \\ \text { K } 3 & 0 & 0 \\ \text { K } 4 & 8 & 8 \\ \text { K } 5 & 21 & 23 \\ \text { K } 6 & 69 & 80\end{array}$

1) Der Anteil der opaken Minerale ist auf 100\% durchsichtige bezogen.

\section{Pollenanalytische Untersuchungen} der Braunkohlenflöze

Obwohl schon mehrfach mikroskopische Untersuchungen an pollenführenden Horizonten des Sylter Kaolinsandes durchgeführt worden sind (F. THIERGart 1944, P. W. Thomson 1948, S. 367, und G. v. D. Brelie \& U. ReIN 1951 in C. Dietz \& H.-L. HECK 1952b, S. 52-53), war eine weitere Bearbeitung notwendig geworden. In den Kaolinsanden treten offensichtlich mehrere Lagen von Braunkohlen bzw. Saprohumolithen in maximaler Mächtigkeit bis zu $1 \mathrm{~m}$ und in geringer Ausdehnung auf. Die pollenanaly- 
tische Untersuchung hatte sich bisher nur auf einzelne Proben aus diesen Horizonten am Roten Kliff und auf unorientierte Braunkohlengerölle vom Strande beschränken müssen. Auch eine weitere, 1954 noch nicht veröffentlichte Pollenanalyse und Ausdeutung von P. W. Thomson (1952) befaßte sich nur mit Einzelproben aus einem im Roten Kliff aufgeschlossenen Flöz.

Es hatten zwar alle Bearbeiter festgestellt, daß die Pollenzusammensetzung in diesen Proben eindeutig auf ein pliozänes Alter der Bildungen hinweist, doch ergab sich durch die im Frühjahr 1954 gewonnene Serie von 10 Proben über $1 \mathrm{~m}$ Flözmächtigkeit am Roten Kliff die Möglichkeit, die Entwicklung der Pollenzusammensetzung in diesem Bildungszeitraum im Diagramm festzuhalten und näher zu studieren. Damit konnten sich vielleicht Einblicke in etwa vorhandene fazielle Unterschiede bei den Braunkohlenund Saprohumolithbildungen sowie Anzeichen für kurzfristige jungpliozäne Klimaschwankungen ergeben. Diese Frage stand im Zusammenhang mit dem gleichfalls mit 10 Proben zu je $10 \mathrm{~cm}$ belegten Flöz im Morsum-Kliff, das bis dahin pollenanalytisch noch garnicht untersucht worden war, und mit dessen Beziehungen zu dem $12 \mathrm{~km}$ entfernten Flöz im Roten Kliff. Schließlich waren in den letzten Jahren die mittel- bis jungpliozänen Ablagerungen (P. W. ThOmson \& H. GreBe 1951), die plio-pleistozänen Grenzschichten (U. ReIN 1951, G. v. D. Brelie \& U. REIN 1952, I. M. vaN DER VLerK \& F. FLorschütz 1953) und die ältesten Interglazialschichten in NW-Deutschland und Großbritannien (H. KarRenberg \& U. Rein 1951, P. W. Thomson in WoldstedT 1951, S. 623-624 und G. LÜTTIG \& U. REIN 1954) an mehreren Lokalitäten bearbeitet worden, so daß sich ein einwandfreier pollenstratigraphischer Vergleich mit den Flözen im Kaolinsand ergeben konnte.

Von dem im Mors u m-K liff freigelegten $1 \mathrm{~m}$ mächtigen Braunkohlenflöz wurden 10 Mischproben über je $10 \mathrm{~cm}$ Profil pollenanalytisch untersucht ${ }^{2}$ ). Die hierbei beobachteten Sporomorphen und ihr prozentualer Anteil an der Zusammensetzung sind für jede Probe innerhalb eines Diagramms (oberer Teil der Abb. 2) zur Darstellung gebracht worden. Es wurden für jede Probe 100 Baumpollen und die auf der gleichen Zählfläche liegenden Nichtbaumpollen bestimmt. Da bei diesen jungtertiären Sporomorphen die Identität bzw. nahe Verwandtschaft mit rezenten Gattungen und Arten gegeben zu sein scheint, wurden bei mehreren Pollentypen die entsprechenden Hinweise hinzugefügt.

Die Zusammensetzung der Sporomorphen ist innerhalb des Flözes verhältnismäßig gleichbleibend. In allen Proben tritt der hohe Anteil an Poll. polyformosus THIERg. (Sequoia), Poll. dubius R. Pot. \& Ven. (Cupressineae) und Poll. serratus R. Рот. \& Ven. (Sciadopitys) als Charakteristikum hervor. Außerdem sind Poll. biatus R. Pot. (Taxodium), Poll. megaexactus R. Рот. (Cyrillaceae), Poll. iliacus und Poll. margaritatus R. Рот. (Ilex), Poll. kruschi R. Por. (Nyssaceae) und Poll. vestibulum R. Рот. (Symplocaceae) in geringen Mengen oder sporadisch vorhanden. Alle genannten Sporomorphen sind wichtige Elemente des jüngsten Tertiärs in Mitteleuropa und werden hier von der Plio-Pleistozängrenze ab, d. h. schon im ältesten Interglazialkomplex von Tegelen, nicht mehr beobachtet. Der Anteil an Poll. microalatus R. Poт., d. h. dem tertiären Pinus haploxylon-Typ (RudolPH), beträgt noch 5 bis $10 \%$, wie dies z. B. für Ablagerungen der pliozänen Reuverstufe üblich ist. Weiterhin vorhandene tertiäre Pollenelemente wie Poll. igniculus R. Рот. (Tsuga diversifolia-Typ (RudolpH) und Poll. viridifluminipites (Wodhouse) = „Spor. macroserratus WolfF" (Tsuga canadensisTyp Rudolph), Poll. simplex R. Pot. \& Ven. (Carya), Poll. stellatus R. Pot. \& Ven. (Pterocarya), Poll. cingulum R. Рот. (Castanea-Typ) und Poll. undulosus Wolff (Ul-

2) Die pollenanalytische Untersuchung wurde im Amt für Bodenforschung, Krefeld, durchgeführt, wobei Fräulein A. MüCKENHAUSEN dankenswerterweise die mikroskopischen Arbeiten übernahm. 

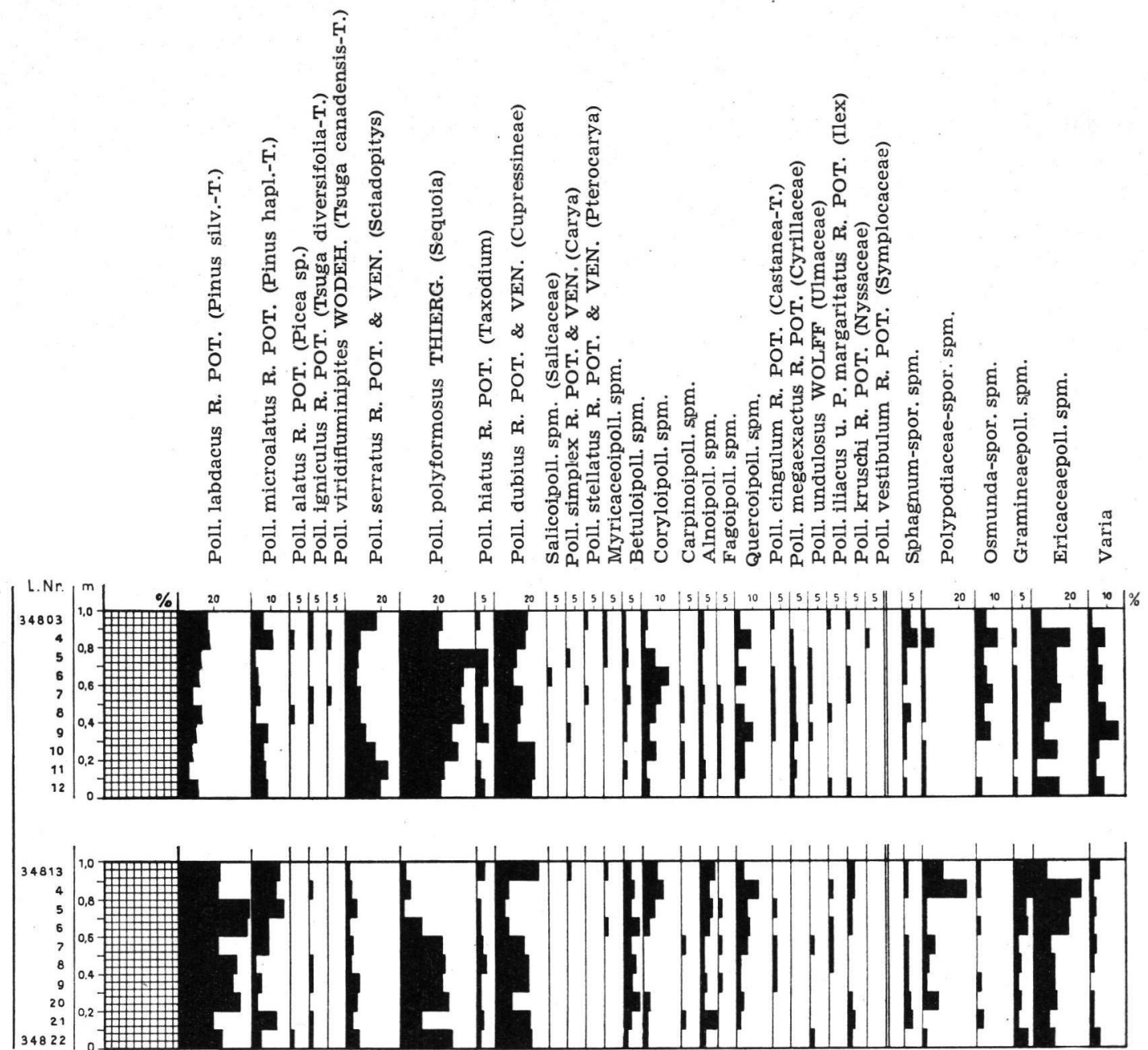

Abb. 2. Diagramm der Pollenzusammensetzung in den Braunkohlenflözen des Kaolinsandes im Morsum Kliff (oberer Teil der Abb.) und im Roten Kliff auf Sylt (unterer Teil der Abb.).

maceae) treten zwar in sehr geringen Mengen noch nach der Pollenverarmung an der Plio-Pleistozängrenze, also im Tegelenkomplex auf, doch ist das relativ zahlreiche und gemeinsame Vorkommen für eine Bildung des jüngeren Pliozäns typisch. Da Fagoipoll. spm. auch noch im Ton von Tegelen (U. ReIN 1950) sowie im Cromer-Interglazial von Bilshausen (G. LütTIG \& U. REIN 1954) gefunden wurde und erst in den jüngeren Interglazialen NW-Deutschlands nicht mehr zurückwandert, ist in der Anwesenheit dieses Pollentyps im Flöz des Kaolinsandes kein genauer stratigraphischer Hinweis gegeben.

Auch im Roten Kliff war zwischen Kampen und Wenningstedt ein $1 \mathrm{~m}$ mächtiges Braunkohlenflöz von der Sturmflut z. T. freigelegt worden. Nach der Anlage eines Schurfes hatte man hier ebenfalls 10 Mischproben entnehmen können. Das Ergebnis der pollenanalytischen Untersuchung ist im unteren Teil der Abb. 2 entsprechend der Lage der einzelnen Proben im Profil als Diagramm aufgetragen worden. Die hier auftretenden Pollentypen sind die gleichen, wie sie im Flöz des Morsum-Kliffs beob- 
achtet und im wesentlichen auch schon von den früheren Bearbeitern der Einzelproben aus dieser und anderen Lagen im Kaolinsand des Roten Kliffs bzw. aus Braunkohlengeröllen vom Strand beschrieben worden sind. Ein Unterschied liegt allerdings gegenüber dem Profil am Morsum-Kliff insofern vor, als der Anteil von Poll. labdacus R. Рот. (Pinus silvestris-Typ) und auch von Poll. microalatus R. Pot. (Pinus haploxylon-Typ) auf Kosten von verringertem Auftreten von Sciadopitys, Sequoia und Cupressineae wesentlich größer ist.

Insgesamt ist das gleiche Bild einer jungpliozänen Pollenzusammens e t z ung zu erkennen, wie es in absolut ähnlicher Ausprägung aus den Ablagerungen der Reuver-Stufe z. B. in stratigraphisch gesicherten Profilen unter dem hangenden „ältesten Diluvialschotter“ aus dem deutsch-holländischen Grenzgebiet von U. REIN (1951), G. v. d. Brelie \& U. Rein (1952) und I. M. v. d. Vlerk \& F. Florschütz (1953) beschrieben worden ist.

Bei einem Vergleich aller bisher bekanntgewordenen Ergebnisse der pollenanalytischen Untersuchung von F. THIERGART 1944 (6 Flözproben), P. W. THOMson 1948 (1 Flözprobe), G. v. d. Brelie \& U. ReIN 1951 (8 Geröllproben) und P. W. Thomson 1952 (1 Flözprobe) mit der vorliegenden Bearbeitung am Roten Kliff zeigt sich über den in allen Fällen herausgestellten jungpliozänen Charakter der Pollenzusammensetzung hinaus eine Vorherrschaft des Pinaceen-Pollens. Gegenüber diesem verhältnismäßig einheitlichen Bild der Pollenführung im Kaolinsand des Roten Kliffs am Westrand von Sylt enthält das Flöz des Morsum-Kliffs einen wesentlich höheren Prozentsatz an Sciadopitys, Sequoia und Cupressineae. Aus Mangel an vergleichbaren Profilen ist z. Z. die Frage noch nicht $z u$ klären, ob hierin nur ein fazieller Unterschied oder auch eine zeitbedingte Differenzierung zu sehen ist. $\mathrm{Da}$ die drei zuletztgenannten Sporomorphen-Typen an der Plio-Pleistozängrenze ganz verschwinden, ist man zunächst eher geneigt, ihr stärkeres Auftreten in den pollenführenden Horizonten am Morsum-Kliff mit einem etwas höheren Alter in Zusammenhang zu bringen.

\section{Zur Petrographie und Fazies der pliozänen Flöze am Roten Kliff und Morsum Kliff auf Sylt}

Bei der pollenanalytischen Untersuchung der im Sylter Kaolinsand auftretenden Flöze durch REIN (s. Abschn. III) hatte sich die Frage ergeben, ob die verschiedene Pollenführung der am Roten Kliff und Morsum Kliff aufgeschlossenen Braunkohlen auf fazielle Unterschiede der Torfbildung zurückgeht.

Aus diesem Grund bat mich Herr Dr. ReIN um eine kohlenpetrographische Untersuchung der für die Pollenanalyse entnommenen Flözproben. $\mathrm{Da}$ es sich hierbei um von $10 \mathrm{zu} 10 \mathrm{~cm}$ entnommene Schlitzproben handelte, konnten die zusammenhängenden $1 \mathrm{~m}$ mächtigen Flözprofile in Form von Stückschliffen nicht untersucht werden. Es wurden daher repräsentative Körnerschliffe der einzelnen Schlitzproben angefertigt und im senkrecht auffallenden Licht mit und ohne Olimmersion studiert. Außerdem wurde von den verschiedenen Faziestypen je ein Dünnschliff untersucht.

\section{Das Flöz am Roten Kliff}

Bei der petrographischen Untersuchung zeigte sich sehr bald, daß man am Roten Kliff von "Kohle“ eigentlich nicht sprechen kann, da das Flö z a u Berorden t lich reich ist an mineralischen Einschwemmungen. Dementsprechend ergab eine Aschenbestimmung der Lage bei 20-30 Flözzentimeter 56,9\% A sche. Der Hauptteil der mineralischen Substanz ist tonig. Schichtweise, besonders bei 20 bis $40 \mathrm{~cm}$ ist stärkere Sandführung zu beobachten. 
Die organische Substanz des Flözes ist vorwiegend humos (vitrinitisch). Sie liegt als feiner Detritus vor, der mit dem Ton innig vermischt ist. Gelegentlich, besonders bei $70-80 \mathrm{~cm}$ und unmittelbar unter dem Hangenden, kommen etwas breitere Vitritlagen (ca. $0,1 \mathrm{~mm}$ ) vor, die in sich vergelt sind und jeweils einem größeren Pflanzenteil (Stengel, Blatt) entsprechen. Fusinit ist sehr selten. Pilzsporen und -gewebe wurden vereinzelt beobachtet. Pyrit kommt nur ganz. untergeordnet in der Lage $80-90 \mathrm{~cm}$ über dem Liegenden vor. Von $80 \mathrm{~cm}$ an aufwärts wurden Einlagerungen eines sehr feinen Tonminerals mit Innenreflexen beobachtet. Wahrscheinlich handelt es sich um reinen Kaolinit.

Das gesamte Flöz zeigt ausgesprochene Mikroschichtung und innige Verzahnung von toniger mit kohliger Komponente. Eine relativ kohlereiche Lage bei $50-60 \mathrm{~cm}$ mit feinster Mikroschichtung erinnert an Kännelkohlen oder ähnliche Schlammablagerungen. Zweifellos ist das gesamte Flöz subaquatisch abgelagert, also eine $\mathrm{Schla} \mathrm{m} \mathrm{mabla-}$ ge r ung und keine Torfbildung im engeren Sinne. Hiermit im Einklang steht der hohe Tongehalt.

Im Gegensatz zu dem Flöz am Morsum Kliff, das großenteils aus einem gewachsenen autochthonen Riedtorf entstanden ist (s. u.), geht die kohlige Substanz des Flözes am Roten Kliff offenbar auf Einschwemmungen von Pflanzendetritus a us der $\mathrm{Nachbarschaft} \mathrm{des} \mathrm{Flözbildungsraumes} \mathrm{zurück.}$

\section{Das Flöz am Morsum Kliff}

Das am Morsum Kliff aufgeschlossene Flöz unterscheidet sich petrographisch und faziell wesentlich von dem Flöz des Roten Kliffs. An Morsum Kliff handelte es sich um Kohle, insofern, als hier die mineralische Komponente stärker zu$\mathrm{rücktritt.} \mathrm{Die} \mathrm{Kurzanalyse} \mathrm{einer} \mathrm{lufttrockenen} \mathrm{Probe} \mathrm{aus} \mathrm{dem} \mathrm{hangenden} \mathrm{Flözteil}$ $(70-80 \mathrm{~cm})$ ergab:

$$
\begin{aligned}
& 10,5 \% \text { Wasser } \\
& 10,9 \% \text { Asche } \\
& 45,2 \% \text { flüchtige Bestandteile }(57,5 \% \text { wasser-, aschefrei) } \\
& 33,4 \% \text { gebundener Kohlenstoff } \\
& \hline 100,0 \%
\end{aligned}
$$

Bei 20-30 Flözzentimeter wurde 18,4\% Asche festgestellt, bei 90-100 Flözzentimeter 18,8\% Asche.

Die mikroskopische Untersuchung der von $10 \mathrm{zu} 10 \mathrm{~cm}$ entnommenen Schlitzproben zeigte deutliche Fazieswechselim Aufbau des Flözes.

Die untersten $10 \mathrm{~cm}$ des Flözes haben großenteils Schwemmto r f - Ch a rakter. Hier finden sich - wohl schon im Torfstadium - umgelagerte eckige Braunkohlenstückchen, größtenteils mit gut erhaltenen humosen Pflanzengeweben. Die Bröckchen sind meist kleiner als $1 \mathrm{~mm}$. Daneben treten Reste von eingeschwemmten, z. T. harzführenden Hölzern auf. Stellenweise sind Harzkörner seifenartig angereichert. Auch andere widerstandsfähige Bestandteile, wie Fusinit und Pilzsklerotien, sind relativ häufig. Der Reichtum an Kutikulen deutet darauf, daß viele Blätter abgelagert wurden. In der Schlitzprobe $0-10 \mathrm{~cm}$ fanden sich neben den Ablagerungen in bewegtem Wasser mit ihren umgelagerten Torf- und Holzstückchen auch Brocken einer feingeschichteten humosen Gyttja mit reichlich Protobitumen. Ein höherer Gehalt an konkretionärem Pyrit im untersten Flözteil deutet auf spätere anaerobe Zersetzungsverhältnisse.

Diefolgenden $10 \mathrm{Flözzentimeter}(10-20 \mathrm{~cm})$ repräsentieren wohleinen humosen Schlamm, derin einem Röhrichtabgelagert wurde. Die Ablagerung ist relativ tonreich, führt viel Protobitumen und zeigt vielfach ausgesprochene Mikroschichtung. Gut erhaltene humose Gewebe treten zurück gegen- 
über einem feinteiligen humosen Detritus. Auffallend ist das Vorkommen toniger Substanz (offenbar Kaolinit) auf Spalten.

Die nächsten 20 Flözzentimeter $(20-40 \mathrm{~cm})$ enthalten praktisch keine zusammenhängenden Pflanzengewebe mehr. Die Kohle besteht hier aus einem äußerst feinen, relativ locker gepackten Detritus, in den feinste Fusinitsplitter und zahlreiche Protobitumen eingebettet sind. Stellenweise sind Pyritkonkretionen häufig und ist eine Feinschichtung angedeutet. Der Tongehalt wird nach oben zu stärker. Zwischen 30 und 40 Flözzentimeter wurden auch umgelagerte Tonbröckchen beobachtet.

Von $40 \mathrm{~cm}$ an aufwärts bis zum Hangenden ist das Flöz petrographisch ziemlich einheitlich aufgebaut. In einem humosen Detritus, der wesentlich gröber ist als der der Lage von $20-40 \mathrm{~cm}$, kommen auffallend viel Fusinit, Resinit u. a. widerstandsfähige Bestandteile (z. B. Pilzsporen) vor. Humose Gewebe treten zurück. In den obersten 20 Zentimetern unter dem Hangenden nimmt der Tongehalt zu, was ja auch aus dem Vergleich der Aschengehalte hervorgeht (bei $70-80 \mathrm{~cm}: 10,9 \%$ Asche, bei $90-100 \mathrm{~cm}$ : $18,8 \%$ Asche). Hier treten stärkere Kaolinpartien auf. Insgesamt dürfte die obere Flözhälfte die Ablagerungen eines Riedmoores repräsentieren.

Nach diesen petrographischen Beobachtungen hat die Flözbildung am Morsum Kliff offenbar mit der Ablagerung verschwemmter Pflanzenteile und Torfstücke begonnen $(0-10 \mathrm{~cm})$. Bald stellten sich Stillwasser-Bedingungen ein, bei denen sich Gyttjen ablagern konnten. Röhricht- und Riedpflanzen siedelten sich an und trugen mehr und mehr zur Schlamm- bzw. Torfbildung bei. Zeitweise, besonders zum Schluß der 'Torfbildung, wurde das Moor in stärkerem Maße überschwemmt, so daß sich reichlich Ton absetzte.

Zur Bildung eines Waldmoores istes offenbar a uch am Morsum Kliff nicht gekommen. Die zahlreichen, von ReIN festgestellten Baumpollen, insbesondere von Sequoia, Taxodium und Sciadopitys müssen eingeweht sein, denn nirgends fanden sich in der Kohle die entsprechenden autochthonen Hölzer, obgleich gerade das Sequoia-Holz besonders widerstandsfähig ist und dort, wo es zur Ablagerung gelangte (wie z. B. vielfach in der Kölner Braunkohle) immer besonders gut erhalten ist.

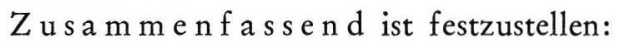

Die am Roten Kliff und Morsum Kliff aufgeschlossenen Flöze unterscheiden sich faziell wesentlich voneinander. Am Roten Kliff ist es zur Bildung eines gewachsenen Torfes nicht gekommen. Das Flöz ist hier allochthon in dem Sinne, daß die pflanzliche Substanz eingeschwemmt wurde. Am Morsum Kliff dagegen haben Röhricht- und Riedpflanzen autochthonen Torf geliefert. Zur Bildung eines Waldtorfes ist es aber auch am Morsum Kliff nicht gekommen. Es ist daher anzunehmen, daß die Polle $\mathrm{n} f \ddot{\mathrm{h}} \mathrm{h}$ r u ng trotz der zahlreichen Pollen von Moorbäumen (Taxodium, Sequoia) auch in der Kohle am Morsum Kliff im wesentlichen ortsfremd ist. Die Unterschiede in der Pollenführung der Flöze am Roten Kliff und Morsum Kliff können also nicht durch die verschiedene Moorfazies erklärt werden.

\section{Schriftenverzeichnis}

BreLIE, G. voN DER \& REIN, U.: Mikropaläontologische Untersuchungen von Braunkohlengeröllen auf der Insel Sylt. - Ber. i. Archiv d. Amtes f. Bodenforsch., Krefeld 1951. Die Interglazialbildungen im niederrheinischen Diluvium. - Der Niederrhein 19, S. 63-68, Krefeld 1952.

Сrommelin, R. D.: Über den Einfluß der nord- und mitteldeutschen Flüsse auf das ältere Pleistozän der Niederlande. - Mitt.Geol.Staatsinst. Hamburg 23, S. 86-97, Hamburg 1954.

Dietz,C. \& Heck, H.-L.: Geologische Karte von Deutschland 1:25000, Bl.Sylt-Nord u. SyltSüd, Kiel 1952 (1952a). - Geologische Karte von Deutschland 1:25 000, Erläuterungen zu den Blättern Sylt-Nord und Sylt-Süd, Kiel 1952 (1952b). 
Karrenberg, H. \& Rein, U.: Die interglazialen Schichten von Krefeld. - Niederrhein. H. Ver. Linker Niederrhein 3, S. 13-17, Krefeld 1951.

LÜtrig, G. \& ReIN, U.: Das Cromer-(Günz/Mindel-)Interglazial von Bilshausen (Unter-Eichsfeld) (Vorläufige Mitteilung). - Geol. Jb. 70, S. 159-166, Hannover 1954.

REIN, U.: Pollenanalytische Untersuchungen zur Pliozän-Pleistozängrenze am linken Niederrhein (Vorläufige Mitteilung). - Geol. Jb. 65, S. 773-778, Hannover 1951.

Thiergart, Fr.: Zur Altersbestimmung eines Saprohumuliths am Roten Kliff auf Sylt zwischen Wenningstedt und Kampen (Buhne 31). Mikropaläontologische Mitteilungen 1-3. Jb. Reichsamt f. Bodenforschung 62, S. 111-114, Berlin 1944.

Thomson, P. W.: Beitrag zur Mikropaläontologie und Waldgeschichte des Neogens (Jungtertiärs) von Niedersachsen und Schleswig-Holstein. - N. Jb. Min. etc. Mh., Jhg. 1945-1948, Abt. B., S. 364-371, Stuttgart 1948. - - Vorläufiger Bericht über die pollenanalytische Untersuchung von vier Proben vom Roten Kliff auf Sylt und Ergänzungsbericht. Ber. i. Arch. d. Amtes f. Bodenforsch., Krefeld 1952. - - Zur Frage des Alters des Braunkohlenlagers vom Roten Kliff auf der Insel Sylt. - N. Jb. Geol. Paläonth., Mh. 1955, 2., S. 68-71, Stuttgart 1955

Thomson, P. W. \& GreBE, H.: Zur Gliederung des tertiären Deckgebirges der rheinischen Braunkohle im südlichen und mittleren Teil der Ville-Scholle und der Erft-Scholle auf mikropaläontologischer Grundlage. - Braunkohle, Wärme und Energie, Jhg. 1951, S. 131-134, Düsseldorf 1951.

VAn der Vlerk, J. M. \& Florschütz, F.: The Paleontological Base of the Subdivision of the Pleistocene in the Netherlands. - Verh. Kon. Ned. Akademie v. Wetensch., Eerste Reeks 20, 2, Amsterdam 1953.

Wetzel, W.: Die Sedimentpetrographie des Sylter Tertiärs. - Schr. naturw. Ver. Schleswig-Holstein 19, Kiel 1931. - - Neue Beobachtungen am Jungtertiär von Sylt. - Jber. nieders. geol. Ver. 28, Hannover 1937.

WEYL, R.: Schwermineraluntersuchungen im schleswigholsteinischen Jungtertiär. - Z.deutsch.geol. Ges. 104, S. 99-133, Hannover 1952. - - Die Schwermineral-Assoziation der Liether Kaolinsande. - Erdöl und Kohle 6, S. 6-7, Hamburg 1953.

Wieseneder, H.: Uber die Veränderung des Schwermineralgehaltes der Sedimente durch Verwitterung und Diagenese. - Erdöl und Kohle 6, Hamburg 1953.

Wirtz, D.: Die Fauna des Sylter Crag und ihre Stellung im Neogen der Nordsee. - Mitt. geol. Staatsinst. Hamburg 19, S. 57-76, 3 Taf,. Hamburg 1949.

Wirtz, D. \& Illies, H.: Plio-Pleistozängrenze und Günzeiszeit in Nordwestdeutschland. - Eiszeitalter und Gegenwart 1, S. 73-83, Öhringen/Württ. 1951 (1951a). - - Lower Pleistocene Stratigraphy and the Plio-Pleistocene Boundary in Northwestern Germany. Journal of Geology 59, S. 463-471, Chicago 1951 (1951b).

WoldstedT, P.: Die Quartärforschung in Deutschland. Ihre Entwricklung und ihre Aufgaben. Z. deutsch. geol. Ges. 100, S. 379-399, Hannover 1950. - - Das Vereisungsgebiet der Britischen Inseln und seine Beziehungen zum festländischen Pleistozän. - Geol. Jb. 65, S.621-640, Hannover 1951.

Manuskr. eingeg. 17. 3. 1955.

Anschriften der Verf.: Dr. Ulrich Rein und Dr. Marlies Teichmüller, Amt für Bodenforschung, Landesstelle Nordrhein-Westfalen, Krefeld, Westwall 124. - Prof. Dr. Richard Weyl, Geolog. Institut der Universität Kiel, Olshausenstraße 40-60. 